\title{
Prognostic Factors for Survival in Patients Treated with Multimodal Therapy for Anaplastic Thyroid Cancer
}

\author{
LUKAS KÄSMANN $^{1}$, LOUISA BOLM $^{1}$, STEFAN JANSSEN $^{1,2}$ and DIRK RADES ${ }^{1}$ \\ ${ }^{1}$ Department of Radiation Oncology, University of Lübeck, Lübeck, Germany; \\ ${ }^{2}$ Medical Practice for Radiotherapy and Radiation Oncology, Hannover, Germany
}

\begin{abstract}
Background/Aim: To identify predictors of survival after multimodal treatment including surgery plus postoperative radio(chemo)therapy) for anaplastic thyroid cancer. Patients and Methods: Nine potential factors were evaluated in nine patients regarding survival after 6, 12 and 24 months. These factors were age, gender, Karnofsky performance score, tumour stage, nodal stage, resection margin status, radiation dose, concurrent chemotherapy administered with irradiation and symptom control at the end of radiotherapy. Results: Survival rates were $67 \%$ at 6 months, $56 \%$ at 12 months and $22 \%$ at 24 months. On univariate survival analysis, concurrent radiochemotherapy $(p=0.018)$ and controlled symptoms at the end of radiotherapy $(p=0.03)$ were associated with improved survival. A trend for better survival was seen in patients with microscopically $(R 1)$ versus macroscopically $(R 2)$ residual disease ( $p=0.058)$. Conclusion: Prognostic factors for survival after multimodal treatment for anaplastic thyroid cancer were identified. Concurrent radio-chemotherapy resulted in significantly better survival and should be recommended.
\end{abstract}

Anaplastic thyroid cancer (ATC) is a rare but very aggressive type of cancer associated with a dismal prognosis (1-7). It represents only $1-5 \%$ of all thyroid tumours. Survival is generally limited to 3-6 months as a consequence of an aggressive tumour biology promoting invasion and metastasis $(1-3,5,8)$. Effective treatment options are

This article is freely accessible online.

Correspondence to: Lukas Käsmann, Department of Radiation Oncology, University of Lübeck, Lübeck, Ratzeburger Allee 160 , D-23538 Lübeck, Germany. Tel: +49 451 5006661; e-mail: LKaesmann@gmail.com

Key Words: Anaplastic thyroid cancer, multimodal treatment, irradiation, outcomes, predictive factors. warranted, and multimodal therapy offers opportunities to prolong survival and improve quality of life (5-7). Curative surgery is a major therapeutic option in the treatment of ATC and the few available data indicate that resection followed by postoperative radiotherapy (PORT) may offer a further survival benefit due to improved local control compared to surgery alone $(2,4)$.

However, there is no standard therapeutic regimen for the treatment of ATC due to lack of evidence. Furthermore, the majority of patients are still treated with palliative intention $(2,6)$. Postoperative PORT combined with concurrent chemotherapy represents an intensive treatment option. It may be associated with high acute toxicity including fatigue, myelopathy, dysphagia or pancytopenia. Consequently, potential candidates for this treatment option should be well selected taking into account the patient's prognosis. However, prognostic factors in the multimodal treatment of ATC remain largely unclear. This study contributes to the treatment allocation and optimization of post-operative radiochemotherapy programs to achieve the best possible treatment outcome. We analyzed nine potential prognostic factors for survival after 6,12 and 24 months.

\section{Patients and Methods}

In this retrospective study, we included nine patients diagnosed with ATC at our hospital from 2006 to 2014 and who underwent multimodal treatment consisting of surgery followed by postoperative radio(chemo)therapy. According to the American Joint Committee on Cancer (AJCC), three patients had stage IVa disease, four patients had stage IVb disease and two patients stage IVc disease, respectively (9). A hemi-thyroidectomy was performed in five patients. In four patients, total thyroidectomy was planned, but only achieved in two patients. Irradiation was performed at median one month after diagnosis of ATC (range: 0-5 months).

Four patients received combined chemotherapy with carboplatin and paclitaxel. Three patients received single-agent chemotherapy with doxorubicin and one patient with gemcitabine. One patient refused the planned concurrent chemotherapy.

The radiation dose was given as equivalent dose in $2 \mathrm{~Gy}$ fractions (EQD2). The median EQD2 was 58 Gy (range: 32.5-60 Gy). Two 
Table I. Patient characteristics and survival analysis (Kaplan-Meier method and the log-rank test).

\begin{tabular}{|c|c|c|c|c|c|}
\hline \multirow[t]{2}{*}{ Characteristic } & \multicolumn{3}{|c|}{ Survival } & \multicolumn{2}{|c|}{$p$-Value } \\
\hline & At 6 months $(\%)$ & At 12 months (\%) & At 24 months (\%) & Univariate analysis & Multivariate analysis \\
\hline \multicolumn{6}{|l|}{ Gender } \\
\hline Female $(\mathrm{n}=2)$ & 50 & 50 & 0 & & \\
\hline Male $(n=7)$ & 71 & 57 & 29 & 0.722 & \\
\hline \multicolumn{6}{|c|}{ Karnofsky performance score } \\
\hline$\leq 80(\mathrm{n}=3)$ & 33 & 33 & 0 & & \\
\hline$>80(\mathrm{n}=6)$ & 83 & 67 & 33 & 0.241 & \\
\hline \multicolumn{6}{|l|}{ Age at radiotherapy } \\
\hline$\leq 64$ years $(n=5)$ & 80 & 80 & 40 & & \\
\hline$\geq 65$ years $(n=4)$ & 50 & 25 & 0 & 0.105 & \\
\hline \multicolumn{6}{|l|}{ Nodal stage } \\
\hline N1a $(n=3)$ & 100 & 100 & 33 & & \\
\hline$>$ N1a $(n=6)$ & 50 & 40 & 17 & 0.192 & \\
\hline \multicolumn{6}{|l|}{ Tumour stage } \\
\hline AJCC IVa $(n=3)$ & 100 & 100 & 33 & & \\
\hline AJCC IVb/c $(n=6)$ & 50 & 40 & 17 & 0.192 & \\
\hline \multicolumn{6}{|l|}{ Resection status } \\
\hline $\mathrm{R} 1(\mathrm{n}=4)$ & 100 & 75 & 50 & & \\
\hline $\mathrm{R} 2(\mathrm{n}=5)$ & 40 & 40 & 0 & 0.058 & 0.096 \\
\hline \multicolumn{6}{|c|}{ Concurrent radiochemotherapy } \\
\hline Yes $(n=7)$ & 86 & 71 & 29 & & \\
\hline No $(n=2)$ & 0 & 0 & 0 & 0.018 & 0.980 \\
\hline \multicolumn{6}{|l|}{ Total radiation dose } \\
\hline$\leq 58$ Gy $(\mathrm{n}=5)$ & 60 & 40 & 0 & & \\
\hline$>58$ Gy $(\mathrm{n}=4)$ & 75 & 75 & 50 & 0.105 & \\
\hline \multicolumn{6}{|c|}{ Symptom control at the end of radiotherapy } \\
\hline Worse $(n=4)$ & 25 & 0 & 0 & & \\
\hline Controlled $(n=5)$ & 100 & 100 & 40 & $\mathbf{0 . 0 3 0}$ & 0.952 \\
\hline
\end{tabular}

AJCC: American Joint Committee on Cancer, R1: microscopically incomplete resection, R2: macroscopically incomplete resection, bold values: significant $p$-values.

EQD2 levels ( $\leq 58$ Gy vs. $>58$ Gy) plus eight additional patient characteristics were analysed for potential associations with survival. These characteristics were age ( $\leq 64 v s . \geq 65$ years), gender, Karnofsky performance score ( $\leq 80 v s .>80$ ), AJCC stage (IVa vs. IVb/IVc), N stage (1a vs. other), resection margin status (microscopically incomplete $=\mathrm{R} 1 \quad v s$. macroscopically incomplete=R2), concurrent chemotherapy (yes vs. no), symptom control at the end of radiotherapy (worse $v s$. controlled symptoms).

The univariate analyses of these factors with respect to survival were performed using the Kaplan-Meier method and the log-rank test (10). Patient characteristics showing a significant association with survival $(p<0.05)$ or at least a trend $(p<0.06)$ were additionally included in a multivariate (Cox regression) analysis.

\section{Results}

Patients were followed until death or for a minimum of 24 months (range $=24-45$ months). Median survival was 12 months (range=2-45 months). The results of the survival analysis are summarized in Table I. Survival rates were $67 \%$ at 6 months, $56 \%$ at 12 months and $22 \%$ at 24 months, respectively. An improved survival at 24 months was found on univariate analysis for concurrent chemotherapy in addition to irradiation $(p=0.018)$ and controlled symptoms at the end of radiotherapy $(p=0.030)$. A trend for better survival was seen in patients after R1resection $v s$. macroscopic R2-resection $(p=0.058)$. None of the investigated factors reached significance on multivariate analysis.

\section{Discussion}

ATC is a highly aggressive tumour with a survival prognosis of only a few months $(1-3,5-8,11)$. For these patients more effective treatment concepts and a set of prognostic factors are needed to optimize outcome and improve treatment allocation. Effective treatment options for patients with ATC are limited. In recent literature, surgery was recommended whenever possible to improve survival and local control. In a retrospective study of 67 patients even incomplete 
resection led to an improvement in survival (4). The first meta-analysis assessing the impact of PORT showed a prolonged survival in patients undergoing surgery followed by PORT when compared to Ipatients treated with surgery alone (2). However, new radiotherapy techniques and radiosensitizing agents plus adjuvant chemotherapy have been suggested to further improve local control and impact survival (8). The use of several anticancer drugs was investigated with more favourable results; however, the patient prognosis remains poor (11).

In a study of Onoda et al. radiochemotherapy with docetaxel improved loco-regional control of patients with ATC and was associated with acceptable toxicity (11). Swaak-Kragten et al. reported an improvement by $23 \%$ in 12-month-survival in patients receiving doxorubicin concurrently with radiotherapy and additionally following radiotherapy (7). However, prognostic factors of survival in this setting still need to be defined. Consequently, the aim of our study was to identify such prognostic factors for patients receiving multimodal treatment in order to optimize treatment allocation and improve individualized patient care. The analysis of the registry of the ATC Research Consortium of Japan showed that the absence of acute symptoms was associated with better survival (12). In our study, symptom control was also a factor significantly associated with improved survival resulting in a 2-year survival rate of $40 \%$. In comparison, recent data from the literature suggested a 2-year survival rate of 10 $20 \%$ (2), whereas Foote et al. reported a 2-year survival rate of $60 \%$ in patients with ATC and favourable patient characteristics such as more favourable tumour stage, namely stage IVa compared to stages IVb and IVc (8). In the study of Foote et al., 70\% of the patients with ATC had stage IVa compared to only $33 \%$ in our present study. This difference may explain the more favourable 2-year survival rate in the study of Foote et al. when compared to the 2year-survival rate in our study (8). Aggressive treatment strategies including surgery, chemotherapy and radiotherapy have been suggested to improve survival in patients with ATC (13). This finding agreed well with the result of our study that the use of concurrent chemotherapy in addition to radiotherapy improved survival. SwaakKragten et al. reported a better local control with concurrent radio-chemotherapy than with radiotherapy alone but no survival benefit (7). In order to validate our findings and to improve the evidence of prognostic factors for patients with ATC, a prospective multicentre study would be required. Furthermore, the optimal chemotherapy program for ATC needs to be defined in prospective studies. However, since patients with ATC are relatively rare, such prospective trials including a cohort of patients large enough to achieve an adequate statistical power cannot be expected soon. Therefore, retrospective studies are the best data currently available. However, with respect to the presents study, its retrospective nature, the relatively small number of patients and the heterogeneous distribution of chemotherapy regimens included should be taken in account.

In conclusion, prognostic factors for survival in patients treated with multimodal therapy for ATC were identified in this study. Concurrent radiochemotherapy resulted in improved survival compared to radiotherapy alone and should, therefore, be recommended, in particular for patients with good performance status.

\section{Conflicts of Interest}

On behalf of all Authors, the corresponding Author states that there is no conflict of interest related to this study.

\section{References}

1 Zivaljevic V, Tausanovic K, Paunovic I, Diklic A, Kalezic N, Zoric G, Sabljak V, Vekic B, Zivic R, Marinkovic J and Sipetic $\mathrm{S}$ : Age as a prognostic factor in anaplastic thyroid cancer. Int $\mathrm{J}$ Endocrinol 2014: 240513, 2014.

2 Kwon J, Kim BH, Jung HW, Besic N, Sugitani I and Wu HG: The prognostic impacts of postoperative radiotherapy in the patients with resected anaplastic thyroid carcinoma: A systematic review and meta-analysis. Eur J Cancer 59: 34-45, 2016.

3 Smallridge RC, Copland J: Anaplastic thyroid carcinoma: pathogenesis and emerging therapies. Clin Oncol 22: 486-497, 2010.

4 Pierie JP, Muzikansky A, Gaz RD, Faquin WC and Ott MJ: The effect of surgery and radiotherapy on outcome of anaplastic thyroid carcinoma. Ann Surg Oncol 9: 57e64, 2002.

5 Sykorova V, Dvorakova S, Vcelak J, Vaclavikova E, Halkova T, Kodetova D, Lastuvka P, Betka J, Vlcek P, Reboun M, Katra $\mathrm{R}$ and Bendlova $\mathrm{B}$ : Search for new genetic biomarkers in poorly differentiated and anaplastic thyroid carcinomas using next generation sequencing. Anticancer Res 35(4): 2029-2036, 2015.

6 Sun C, Li Q, Hu Z, He J, Li C, Li G, Tao X and Yang A: Treatment and prognosis of anaplastic thyroid carcinoma: experience from a single institution in China. PLoS One $5 ; 8(11)$ : e80011, 2013.

7 Swaak-Kragten AT, de Wilt JH, Schmitz PI, Bontenbal M and Levendag PC: Multimodality treatment for anaplastic thyroid carcinoma-treatment outcome in 75 patients. Radiother Oncol 92: 100-104, 2009.

8 Foote RL, Molina JR, Kasperbauer JL, Lloyd RV, McIver B, Morris JC, Grant CS, Thompson GB, Richards ML, Hay ID, Smallridge RC and Bible KC: Enhanced survival in locoregionally confined anaplastic thyroid carcinoma: a singleinstitution experience using aggressive multimodal therapy. Thyroid 21: 25-30, 2011.

9 Edge SB and Compton CC: The American Joint Committee on Cancer: the 7th edition of the AJCC cancer staging manual and the future of TNM. Ann Surg Oncol 17: 1471-1474, 2010.

10 Kaplan E and Meier P: Nonparametric estimation from incomplete observation. J Am Stat Assoc 53: 457-481, 1958. 
11 Onoda N, Kashiwagi S, Noda S, Kawajiri H, Takashima T, Ishikawa $\mathrm{T}$ and Hirakawa K: High efficacy of chemoradiation therapy sensitized by weekly docetaxel for anaplastic thyroid cancer. Anticancer Res 33: 3445-3448, 2013.

12 Sugitani I, Miyauchi A, Sugino K, Okamoto T, Yoshida A and Suzuki S: Prognostic factors and treatment outcomes for anaplastic thyroid carcinoma: ATC Research Consortium of Japan cohort study of 677 patients. World J Surg 36: 1247-1254, 2012.
13 Nachalon Y, Stern-Shavit S, Bachar G, Shvero J, Limon D and Popovtzer A: Aggressive Palliation and Survival in Anaplastic Thyroid Carcinoma. JAMA Otolaryngol Neck Surg 141: 11281132, 2015.

Received July 20, 2016

Revised August 5, 2016

Accepted August 18, 2016 